\title{
Proximity requirements for auditory gap detection'
}

\author{
IRWIN POLLACK ${ }^{2}$ \\ THE UNIVERSITY OF MICHIGAN
}

The discrimination of a single temporal discontinuity, or gap, within an auditory pulse train was studied as a function of the relative magnitude of the gap region to its immediately surrounding interpulse intervals (the near surround) and to interpulse intervals flanking the near surround (the far surround). Near-surround effects substantially outweigh far-surround effects.

One test of temporal acuity is provided by the "gap" test-the detection of a single temporal discontinuity (Pollack, 1967; Uttal \& Krossoff, 1966) or the relative discrimination among a series of temporal discontinuities (Pollack, 1969). The acuity of gap detection clearly depends upon the

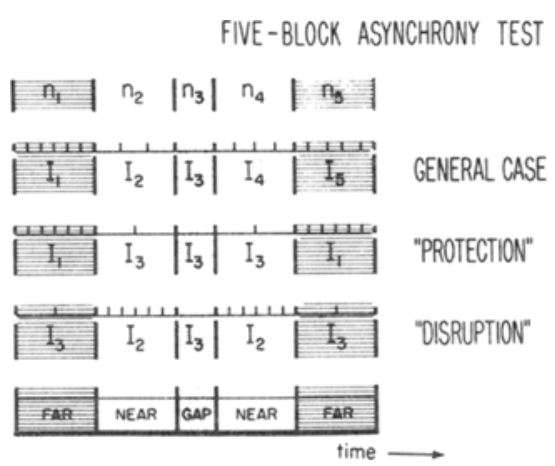

Fig. 1. Schematic illustration of near and far surrounds about a reference-gap region.

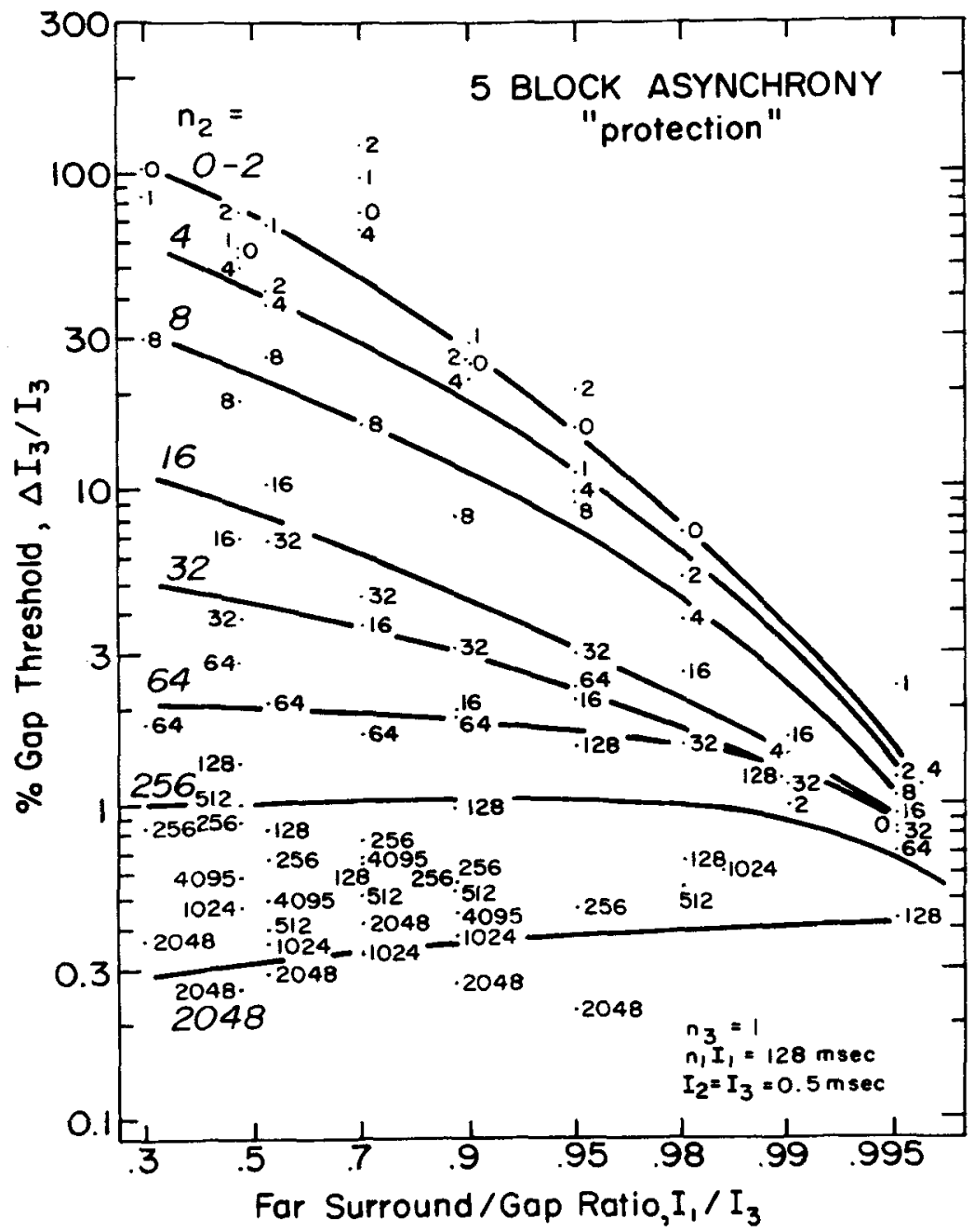

interpulse intervals that surround the gap. If a temporal discontinuity is inserted within an otherwise periodic sequence, gap-detection thresholds may be substantially smaller than if the gap is inserted within an irregular or jittered sequence of intervals (Pollack, 1968). Relative gap discrimination, i.e., the discrimination between two different gaps, may also suffer, even within strictly periodic sequences, when the reference gap differs from its surrounding intervals (Pollack, 1969). The results thus suggest that gap sensitivity may be extremely sensitive to the temporal microstructure in the immediate vicinity of the gap. The present paper attempts to specify more fully the local temporal determinants related to gap detection and to relative gap discrimination.

\section{METHOD}

The basic paradigm is illustrated schematically in the top line of Fig. 1. A pulse train consists of five blocks of interpulse intervals: $I_{1}, I_{2}, I_{3}, I_{4}$, and $I_{5}$, with $n_{1}, n_{2}, n_{3}, n_{4}$, and $n_{5}$ interpulse intervals, respectively. The middle region, $I_{3}$, is defined as the gap region. The gap region of $n_{3}$ intervals is immediately flanked by a near surround of $n_{2}$ pre-gap intervals and $n_{4}$ post-gap intervals. The near surround is, in turn, flanked by a far surround of $n_{1}$ pre-gap intervals and $n_{5}$ post-gap intervals.

Two cases of symmetrical detection are illustrated in the second and third lines of Fig. 1. In a previous study, with a threesection paradigm $\left(n_{2}, n_{3}, n_{4}\right.$, and $I_{2}, I_{3}$, $I_{4}$ of Fig. 1), it was shown that relative gap discrimination increasingly suffered when the immediate surround, $I_{2}$ and $I_{4}$, increasingly departed from the reference gap, I 3 (Pollack, 1969). The second line considers the "protection" of gap sensitivity by a near surround, identical to the gap region, against a nonidentical far

Fig. 2. Protection of gap sensitivity afforded by an identical near surround. The abscissa is the ratio of the far-surround intervals to the reference gap; the parameter is the number of near-surround intervals protecting the reference gap. $T$ wo sets of points are listed at an $I_{1} / \Lambda_{3}$ ratio of 0.5 . The left set was obtained at a ratio of 0.5 ; the right set was obtained at a ratio of 2.0. 


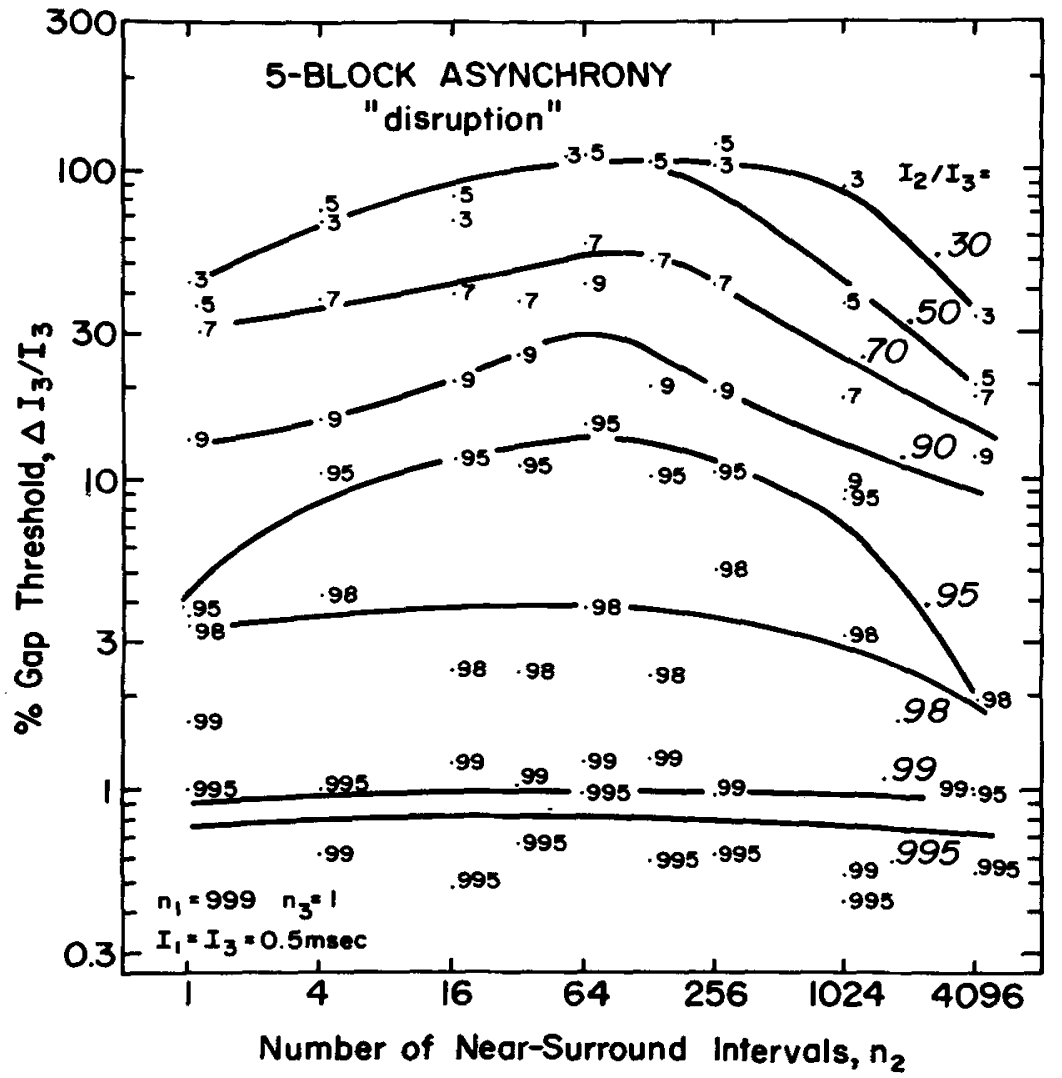

surround. The third line considers the "disruption" of gap sensitivity by a nonidentical near surround interposed between the gap region and an identical far surround. The term, "identical," here refers to the relation of the surround interval to that of the reference-gap region.

Unless specified otherwise, the following restrictions were observed: $n_{3}=1, n_{1}=n_{5}$, $\mathrm{n}_{2}=\mathrm{n}_{4}, \mathrm{I}_{1}=\mathrm{I}_{5}, \mathrm{I}_{2}=\mathrm{I}_{4}, \Delta \mathrm{I}_{3} \geqslant 0, \mathrm{I}_{1} \leqslant \mathrm{I}_{3}$, $I_{2} \leqslant I_{3}$, i.e., interference was primarily examined for shorter upon longer intervals.

Pulse trains were generated by a PDP-8 (Digital Equipment Corp.) computer. The unit of temporal control was $0.375 \mu \mathrm{sec}$. Brief pulses $(10 \mu \mathrm{sec})$ were smoothed by binaural earphones (Koss PRO-4). The pulse trains were adjusted to a comfortable listening level. In a four-interval forced-choice (4IFC) discrimination test, three standard pulse trains and one variable pulse train were presented. All four trains were identical, except that $I_{3}$ was incremented by $\Delta I_{3}$ for the variable pulse train. The task of the listener was to identify whether the first, the second, the third, or the fourth train contained the temporal gap. An adaptive stimulus-programming procedure of variable step size (Taylor \& Creelman, $1967)$ varied $\Delta I_{3}$ to converge upon $50 \%$ correct response in the 4IFC test. Each point of Figs. 2-7 represents at least two thresholds by each of 15-18 listeners. Gap sensitivity will be expressed relative to the reference gap, $\Delta \mathrm{I}_{3} / \mathrm{I}_{3}$.

\section{"Protection" by an Identical Near Surround}

The tests of Fig. 2 sought to answer whether or not gap sensitivity was "protected" by an identical near surround interposed between the reference gap and nonidentical far surround. In these tests, the near surround and the reference gap intervals were held constant at $0.5 \mathrm{msec}$, and the interpulse interval of the far surround, $\mathrm{I}_{1}$, was varied.

The main point of Fig. 2 is that gap sensitivity varies over nearly a three-decade range as the number of near-surround intervals is varied. There is a suggestion that gap thresholds may be smaller with zero near-surround intervals than with a small number of intervals. The effect of the interval discrepancy between the far surround and the reference gap is related to the number of near-surround intervals; the $I_{1} / I_{3}$ ratio associated with a large change in sensitivity only with a small number of near-surround intervals. With a large number of near-surround intervals, only a small change in sensitivity is associated with the discrepancy between the far surround and the reference gap. The
Fig. 3. Disruption to gap sensitivity associated with a nonidentical near surround. The abscissa is the number of near-surround intervals; the parameter is the interval-ratio discrepancy between the near surround and the gap region.

results are consistent with the conclusion that gap sensitivity is primarily determined by the immediate temporal environment of the gap region.

\section{"Disruption" by a Nonidentical Near Surround}

The tests of Fig. 3 sought to answer whether or not gap sensitivity was "disrupted" by a nonidentical near surround interposed between the reference gap and an identical far surround. In these tests, the far-surround and the reference-gap intervals were held constant at $0.5 \mathrm{msec}$, and the interpulse interval of the near surround, $I_{2}$, was varied.

The main point of Fig. 3 is that the disruption produced by a nonidentical near surround is extremely sensitive to the interval discrepancy between the near surround and the gap region. For $I_{2} / I_{3}$ ratios of 0.98 and higher, gap sensitivity is nearly independent of the number of nonidentical near-surround intervals; for smaller ratios, gap sensitivity is somewhat poorer for an intermediate number of near-surround intervals and improves for both a smaller number, and for a larger number, of nonidentical near-surround intervals. The striking result is that only a single nonidentical near-surround interval produces nearly the full disruption effect. With $n_{1}=999$, the equivalent threshold with zero nonidentical near-surround intervals is about $0.3 \%$. Therefore, only a single nonidentical near-surround interval serves to raise the gap threshold by over a 100-fold.

\section{Relative Contributions by the Near and Far Surrounds}

The tests of Fig. 4 sought to answer how gap sensitivity varies with the extent of the near and far surrounds. In these tests, the near-surround intervals and reference gap were held constant at $0.5 \mathrm{msec}$; the far-surround intervals were held constant at $0.25 \mathrm{msec}$, and the number of near and far surround intervals was varied.

The main point of Fig. 4 is that gap detection varies over nearly a three-decade range of sensitivity with changes in the number of identical near-surround intervals. Gap thresholds are, however, relatively independent of the number of far-surround intervals, with somewhat larger thresholds for an intermediate number of nonidentical far-surround 

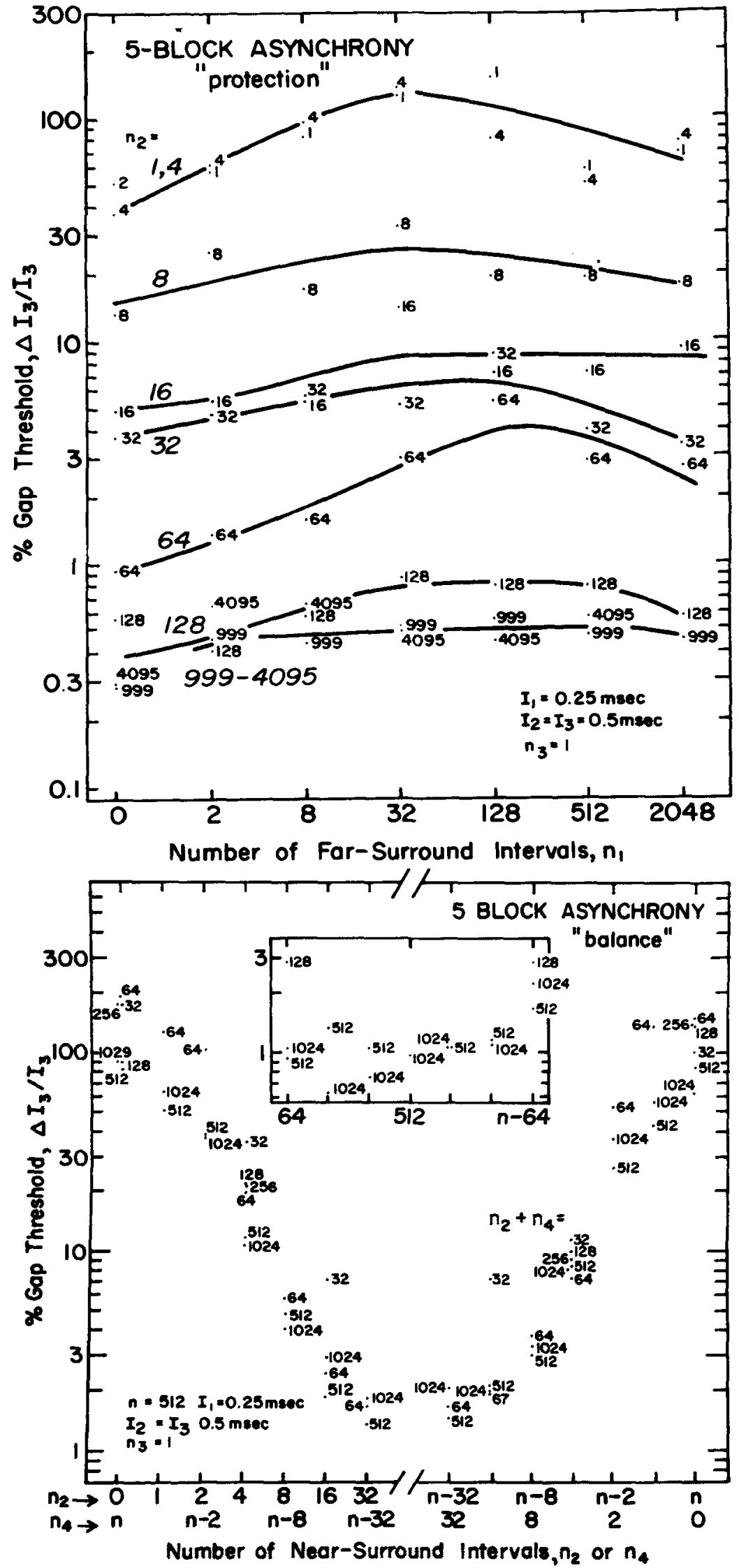

Fig. 4. The relative lack of contribution associated with a nonidentical far surround. The abscissa is the number of nonidentical far-surround intervals; the parameter is the number of identical near-surround intervals.

intervals than a larger or smaller number of intervals. This result suggests again that gap sensitivity is tightly tied to the temporal microstructure only in the immediate temporal region of the gap.

Interaction of the Protective Effects of Pre-Gap and Post-Gap Near-Surround Intervals

The tests of Fig. 5 sought to determine how the protection of gap sensitivity depends upon the distribution of identical intervals in the near-surround region. In these tests, the protection paradigm of Fig. 2 is extended to asymmetrical near surrounds, i.e., with $\mathbf{n}_{2} \neq \mathbf{n}_{4}$.

The main point of Fig. 5 is that gap thresholds are determined primarily by the smaller of the pre-gap and post-gap surrounds. For a given number of pre-gap or post-gap intervals, gap sensitivity is largely independent of the total number of surround intervals. This result supports the conclusion of Fig. 4, in which gap sensitivity was related to the temporal microstructure only in the immediate region of the gap.

\section{Extension to Wider Gap Regions}

The tests of Fig. 6 sought to determine how the protection of gap sensitivity depends upon the extent of the gap region. In these tests, the protection paradigm of Fig. 2 is extended to gap regions of more than one interval, i.e., $\mathrm{n}_{3}>1$.

The main point of Fig. 6 is that the relative protection afforded by an identical near surround is substantially greater with a single-interval gap region than for an. extended interval gap region. A two-decade: change in gap sensitivity obtains with $\mathrm{n}_{3}=1$; less than a one-decade change in gap sensitivity obtains with $n_{3}=256$. With a still larger number of intervals in the gap region, pitch or interval discrimination might be anticipated to be independent of the surround.

\section{Extension to Different Gap Intervals}

The tests of Fig. 7 sought to determine

Fig. 5. Interaction of pre-gap and post-gap identical near-surround intervals. The abscissa represents the number of pre-gap and the number of post-gap identical near-surround intervals. The parameter is the total number of near-surround intervals. The insert represents an extension to a still Iarger number of near-surround intervals. 

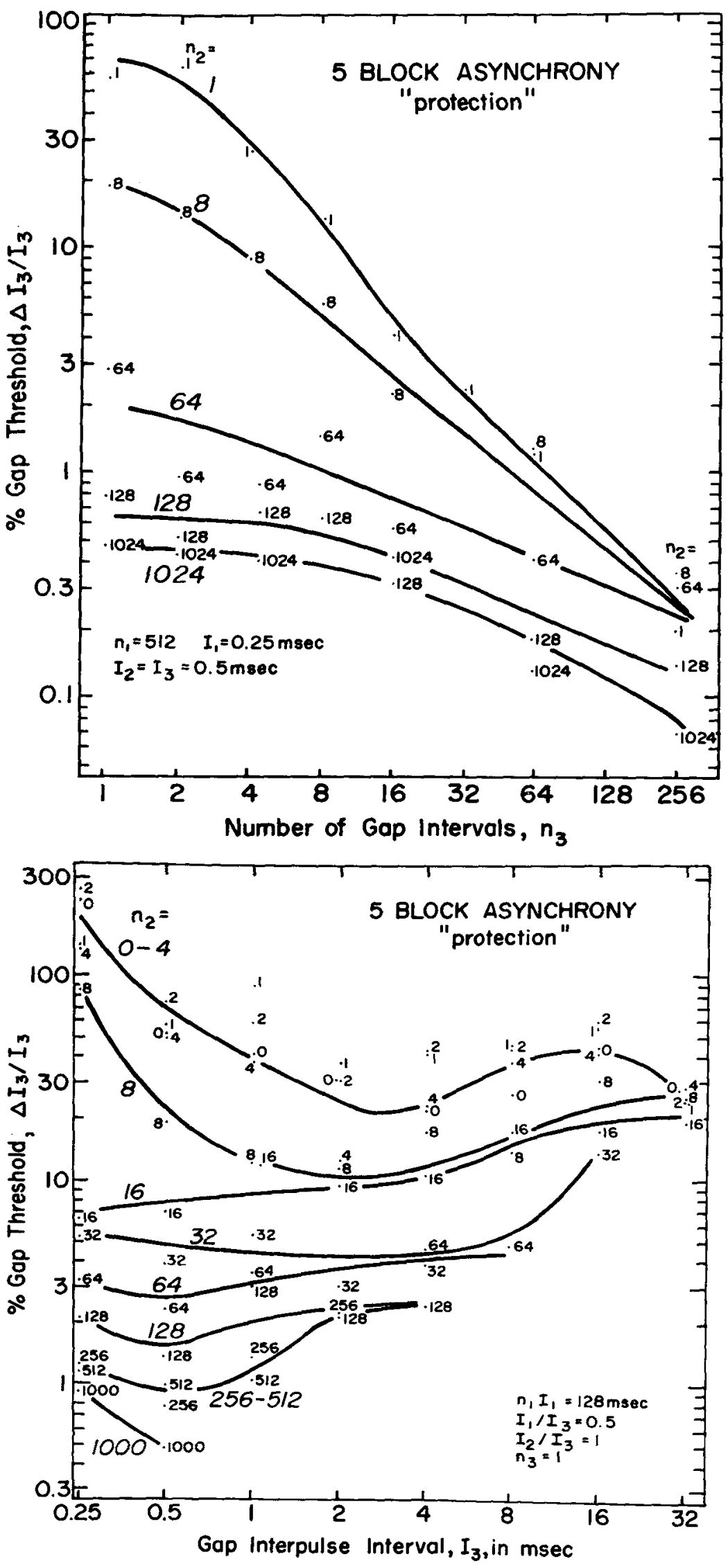

Fig. 6. The diminishing effect of the near surround with extended gap regions. The abscissa is the number of gap intervals; the parameter is the number of identical near-surround intervals.

the range of pulse frequencies over which "protection" by an identical near surround is effective. In these tests, the protection paradigm of Fig. 2 is extended to a range of reference-gap intervals. A far surround $\mathrm{n}_{1} \mathrm{I}_{1}$ of $128 \mathrm{msec}$ with $\mathrm{I}_{1} / \mathrm{I}_{3}$ ratio of 0.5 flanked $\mathrm{n}_{2}$ identical near-surround intervals.

The main point of Fig. 7 is that larger changes in gap sensitivity, as a function of the number of near-surround intervals, are obtained at the higher pulse frequencies (short IPIs) than at lower pulse frequencies. The change results both from a loss of gap sensitivity with a small number of identical near-surround intervals and an improvement in gap sensitivity with a large number of identical surround intervals at high pulse frequencies.

\section{DISCUSSION}

The outstanding result is the extreme dependence of gap-interval detection upon the near surround and the relative independence of the far surround. Gap thresholds change by a factor of more than $300: 1$ as a function of the number of identical near-surround intervals, irrespective of the number and size of the far-surround intervals. Similarly, a single nonidentical near-surround interval, interposed between the reference gap and the far surround, produces nearly complete disruption. Additional nonidentical near-surround intervals produce little further effects.

The gap tests thus seem to be measuring extremely isolated local effects in the gap region. Since the various effects become attenuated at lower and lower pulse frequencies, it is tempting to consider that the limits for gap detection are laid down by the requirements for a spectral analysis of the gap region.

\section{APPENDIX: ASSOCIATED STUDIES}

Two associated series of tests serve to extend the findings of the main study.

Double-gap tests. In the main tests, a single temporal discontinuity occurred within each of four pulse trains. The listener made his judgment upon some

Fig. 7. Protection of gap sensitivity afforded by an identical near surround for various reference-gap intervals. The size of the reference-gap interval is represented on the abscissa; the parameter is the number of near-surround intervals on each side of the gap region. 

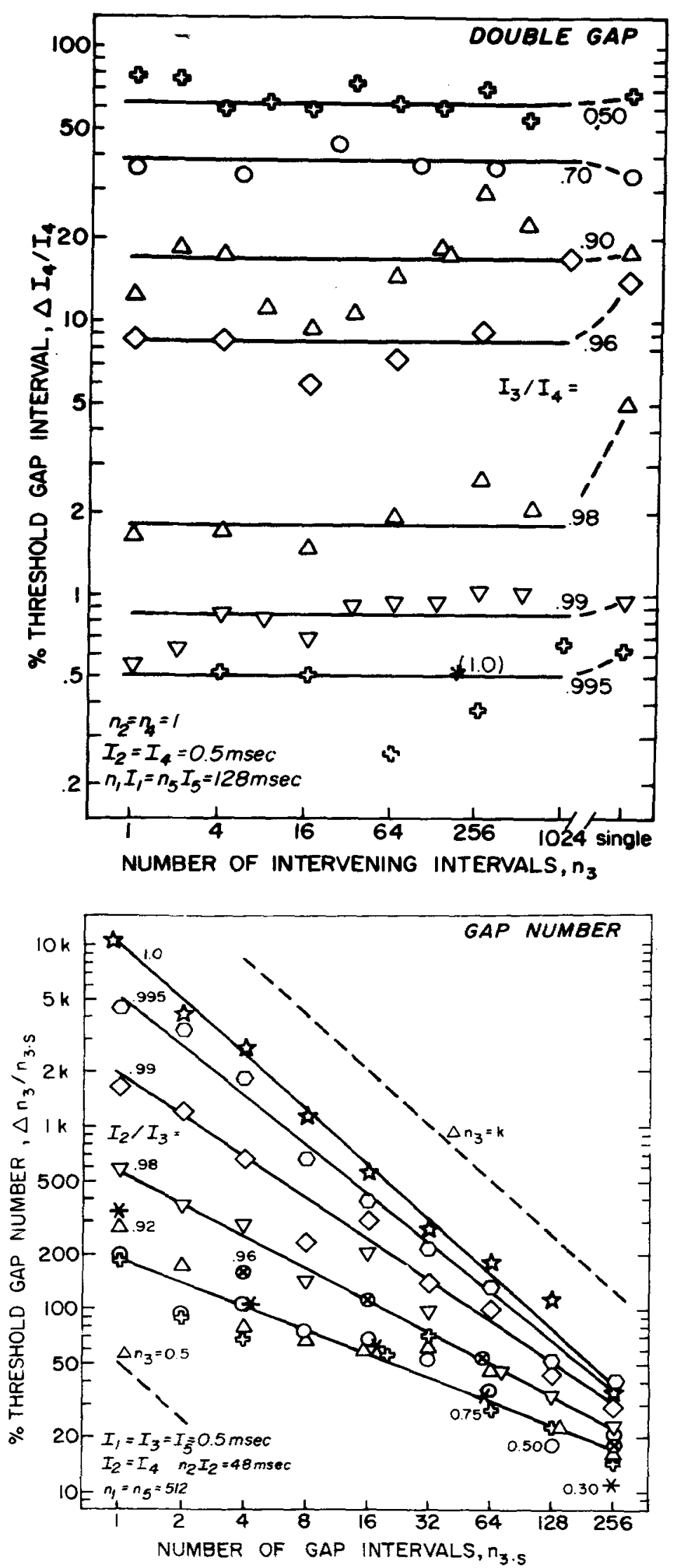

Fig. A 1. Gap interval thresholds for the double gap procedure. The points on the right side of the figure represent gap interval thresholds in single gap tests with a neas surround of $128 \mathrm{msec}$. The parameter is the $I_{1,3,5} / I_{2,4}$ interval ratio.

comparison among the four discontinuities. Additional tests were constructed in which each pulse train had two gaps. With $I_{1}=I_{3}=I_{5}$, a reference gap, $I_{2}$, was common to all pulse trains. In three of four pulse trains, a second reference gap was presented, $I_{4}=I_{2}$. In one of the four pulse trains, a variable gap was presented $I_{4}=I_{2}+\Delta I$. In this manner, the listener could compare two gaps within the same pulse train. Does this procedure buy appreciable gains in gap-interval sensitivity? Apparently not.

The results of the double-gap tests are shown in Fig. A1. The abscissa is the number of $n_{3}$ intervals intervening between $I_{2}$ and $I_{4}$. The right-most points were taken from single-gap tests in which the near surround was the same duration as $n_{1} I_{1}$ and $n_{5} I_{5}$. The parameter is the interval ratio $I_{1,3,5} / I_{2,4}$. The major points are that the double-gap threshold closely follows the single-gap threshold, with a possible improvement in the region of interval ratios of $0.96-0.98$. And, the double-gap threshold is virtually independent of the number of intervening intervals. Other tests with a larger number of $n_{2}$ and $n_{4}$ intervals show, again, that double-gap thresholds are independent of the number of intervening intervals, $n_{3}$, but, as in Fig. 6, are sensitive to the number of gap intervals.

Gap-number tests. In the main tests, the number of gap intervals in the reference and variable trains was equal. Consider now the case of discrimination of the number of gap intervals. If the gap intervals are clearly differentiated from their background, discrimination of the number of gap intervals might be expected to be independent of the background. If the gap intervals are completely indistinguishable from their background, discrimination of the number of gap intervals might be expected to depend upon the total number of intervals. Thus, a measure of the distinctiveness of the gap region is the discrimination of the number of gap intervals.

The single-gap "disruption" paradigm of Fig. 1 was used. The experimental variable was $n_{3 . v}$, the number of intervals in the gap region of the variable signal.

The major results are presented in Figs. $A 2$ and A3. Figure A2 plots the relative gap-number threshold as a function of the

Fig A2. Gap number differential thresholds as a function of the number of gap intervals in the reference pulse train. The slope of the dashed lines represents a constant $\Delta \mathbf{n}_{3}$ threshold. The parameter is the $I_{2,4} / I_{1,3,5}$ interval ratio. 


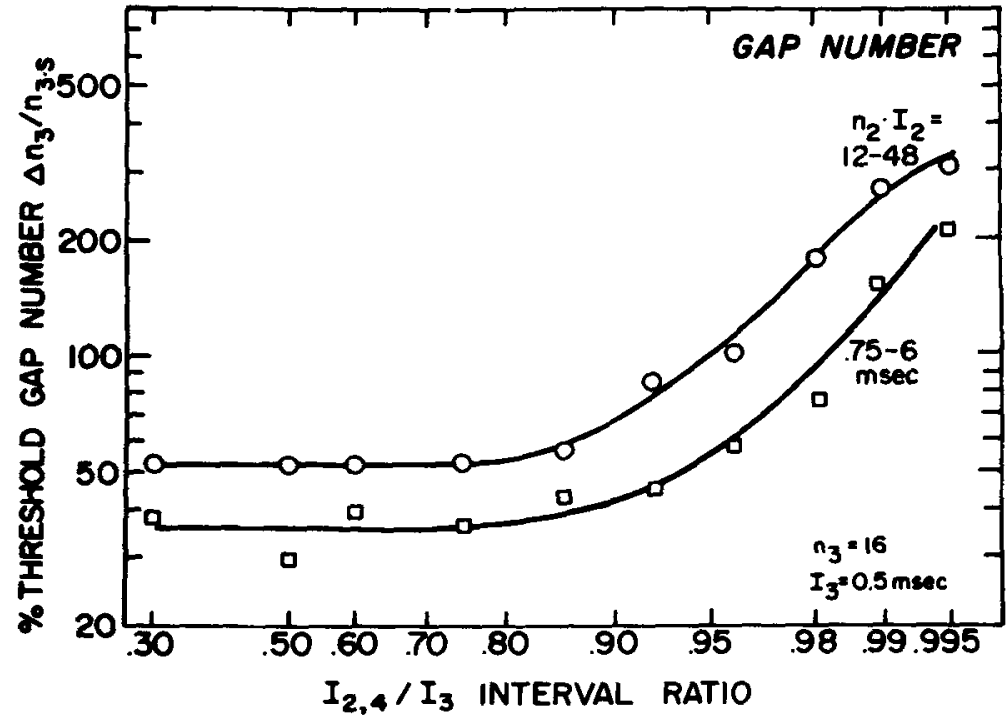

number of gap intervals in the reference train. The parameter is the $I_{2} / I_{3}$ interval ratio. At an $I_{2} / I_{3}$ interval ratio of 1.0 , the gap and near-surround intervals are identical. Number discrimination must be based upon the total number of intervals $\left(n_{1}=n_{5}=512 ; \quad n_{2}=n_{4}=192 ;\right.$ total $=$ $\left.1408+n_{3}\right)$. Thus, a discrimination threshold of $11,000 \%$ for $n_{3} s=1$ implies a threshold $\Delta \mathrm{n}$ of 110 from a total of 1,409 intervals, or about $8 \%$. The upper dashed line is the slope of a constant $\Delta n_{3}$, expressed in terms of the differential threshold, relative to the number of reference-gap intervals. The gap-number discrimination function approaches the limiting slope for an $I_{2} / I_{3}$ interval ratio of 1.0. As the interval departs more and more from unity, the gap-number thresholds decrease at least to an interval ratio of about 0.90. However, even for extreme interval ratios, the differential threshold does not approach pulse-number thresholds of the order of $10 \%$ without a surround (Pollack, Journal of Experimental Psychology, 1968, 78, 660-6C5) or the lower limit available to the program, $\Delta \mathrm{n}=0.5$ count. Figure A3 suggests that gap-number thresholds improve by a factor of about 2.0 as the near surround is more precisely marked by decreasing its duration.
Fig. A3. Gap number differential thresholds as a function of the gap/near-surround interval ratio: The top set of points represent $n_{2} I_{2}$ intervals between 12 and $48 \mathrm{msec}$; the bottom set of points represent $n_{2} I_{2}$ intervals between 0.75 and $6 \mathrm{msec}$.

\section{REFERENCES}

POLLACK, I. Asynchrony: The perception of temporal gaps within auditory pulse patterns. Journal of the Acoustical Society of America, $1967,42,1335-1340$

POLLACK, I. Asynchrony: II. Perception of temporal gaps within periodic and jittered pulse patterns. Journal of the Acoustical Society of America, 1968, 43, 7476.

POLLACK, I. Differential asynchrony thresholds for auditory pulse trains. Journal of the Acoustical Society of America, 1969, 45, 446-449.

TAYLOR, M. M., \& CREELMAN, C. D. PEST Efficient estimates on robability functions. Journal of the Acoustical Society of America. $1967,41,782-787$.

UTTAL, W, R, \& KRISSOFF, M. Effect of stimulus pattem on temporal acuity in the somatosensory system. Journal of Experimental Psychology, 1966, 71, 78-883.

\section{NOTES}

1. This research was supported in part by NSF Grant GB 6148. The author is indebted to Miss Ann Palmer, who developed the computer program, and to Mrs. Dorothy LaBarr, who supervised the experimental tests.

2. Address: Mental Health Research Institute, The University of Michigan, Ann Arbor, Michigan 48104 .

(Accepted for publication May 15, 1969.) 